\title{
Tribological Classification of Contact Lenses: From Coefficient of Friction to Sliding Work
}

\author{
O. Sterner ${ }^{1} \cdot$ R. Aeschlimann ${ }^{1} \cdot$ S. Zürcher ${ }^{1,2} \cdot$ C. Scales ${ }^{3} \cdot$ D. Riederer ${ }^{3}$. \\ N. D. Spencer ${ }^{2}$ S. G. P. Tosatti ${ }^{1}$
}

Received: 29 January 2016/ Accepted: 11 May 2016/Published online: 21 May 2016

(c) The Author(s) 2016. This article is published with open access at Springerlink.com

\begin{abstract}
The coefficient of friction (CoF) has been reported to correlate with clinical comfort of soft contact lenses (SCL). However, a classification in terms of a $\mathrm{CoF}$ is not always applicable to soft materials, such as hydrogels, due to the frequently observed nonlinearity between the lateral and the normal forces. An alternative methodology is presented to quantify the tribological characteristics of soft materials under boundary lubrication in terms of average work. Average work was derived from knowledge of the area of contact, the interfacial shear stress, and sliding distance. To illustrate the work concept, three commercially available SCL $(n=10)$ and rabbit corneas $(n=10)$ were characterized with regard to lateral force against a biomimetic mucin-coated glass disk in a tear-like fluid, by means of microtribometry. The contact area between the glass disk and the SCL was measured in situ and fitted to an elastic-foundation model of the material. On the cornea, the contact area was observed via the expulsion of a fluorescent marker from the contact region. All SCL materials had significantly $(p<0.05)$ different values for average work. Furthermore, the interfacial shear stress on the cornea was found to be at least an order of magnitude lower than on any of the SCL. Average work
\end{abstract}

Electronic supplementary material The online version of this article (doi:10.1007/s11249-016-0696-5) contains supplementary material, which is available to authorized users.

S. G. P. Tosatti

samuele.tosatti@susos.com

SuSoS AG, Lagerstrasse 14, 8006 Duebendorf, Switzerland

2 Laboratory for Surface Science and Technology, Department of Materials, ETH Zurich, Vladimir-Prelog-Weg 5, 8093 Zurich, Switzerland

3 Johnson \& Johnson Vision Care Inc., Jacksonville, FL, USA represents a single figure of merit for the lubricious properties of soft materials, such as SCL, that do not show a linear relationship between lateral and normal forces.

Keywords Contact lens · Friction · Hydrogel ·

Viscoelastic material · Cornea $\cdot$ Frictional energy

\section{Introduction}

A crucial aspect of the eye's blinking cycle is the low frictional losses that the eyelid experiences as it glides back and forth across the cornea. The two soft epithelia are supported by tear films-a complex lubricant system consisting of a stratified, lipid-covered protein mixture, whose structure is broken and reformed during the opening and closing phase of a blink $[1,2]$. The lid-wiper region of the palpebral conjunctiva travels at speeds that can reach $12 \mathrm{~cm} / \mathrm{s}$, suggesting a primarily hydrodynamic lubrication regime. Thus, during the majority of a blink cycle, the sliding resistance is governed by the viscous shear of the lubricant [3]. At the reversal points, where the speed invariably approaches zero, the glycocalyx and associated mucus layer ensure low interfacial shear stresses, which can minimize strain-induced wear of the epithelium [4, 5].

The presence of a soft contact lens (SCL) between the cornea and the eyelid disrupts the natural function of the tear-film, leading to changes in the tear-exchange rate, and in the stability and activity of lipids and proteins in the lubricant as they interact with the lens surface [6-8]. In addition, the contact-lens surface naturally lacks a glycocalyx, which may lead to increased strain on the eyelid epithelium during the reversal points of the blink cycle. SCL wearers may experience complications due to these effects, with changes in comfort being the most common 
reason for interrupted wear [9]. There is evidence that comfort of SCLs is correlated with the frictional properties of the lens material $[10,11]$. In this context, the coefficient of friction of fresh human corneas (measured within $12 \mathrm{~h}$ post-mortem) against a mucin-coated glass disk in a physiologically relevant, tear-like fluid (TLF) has been measured and was reported to lie between 0.006 and 0.015 [12]. As shown by Roba et al. [13] under similar conditions (with a lubricant that contained only serum and lysozyme, with no added lipids), the $\mathrm{CoF}$ of a number of commercially available SCL falls within, or even below, this range. This would suggest that some SCL would be completely exempt from issues related to comfort. However, it is well known that SCL accumulate proteins and lipids, both at the surface and in the bulk, depending on the SCL material, which leads to changes in surface properties of the pristine lens-a topic recently reviewed by Luensmann and Jones [14]. Indeed, a recent report revealed that some SCL that underwent an in vitro aging process, consisting of constant immersion and withdrawal from TLF for $18 \mathrm{~h}$, had an increase in $\mathrm{CoF}$ [15]. This indicates that the frictional losses during blinking may build up over a day's wear, increasing the energy expended by the eyelid to overcome the dynamic friction. The correlation between comfort and $\mathrm{CoF}$, and the possibility of predicting the performance of SCLs from in vitro experiments, has led to numerous studies dealing with the characterization of the frictional properties of SCLs [16-21].

Characterizing the tribological behavior of soft materials, such as a hydrated SCL or rubbers, by a CoF, can be useful to a first approximation. However, the linear dependence between frictional and normal forces that is assumed when calculating a $\mathrm{CoF}$ is frequently not observed in hard-soft and soft-soft contacts [22-24]. The mechanical origin of the linearity between frictional and normal forces has been suggested to arise from the asperity-asperity contacts that define the real area of contact between two hard surfaces. With increasing normal load, an increasing number of asperities comprises the contact area, resulting in a linear dependence between normal force and real contact area [25, 26]. In contrast, Hertzian contact mechanics predicts that the contact area varies with $F_{N}^{2 / 3}$ [27]. For soft materials, asperities are likely to completely deform, and the real contact area can approach the apparent value, resulting in a deviation from linearity between friction and normal forces [28]. SCL typically have elastic moduli of or below $1 \mathrm{MPa}$ [29], and as a consequence, the $\mathrm{CoF}$ may not adequately describe the tribological characteristics over different normal loads.

In this publication, an alternative strategy is proposed to characterize the friction behavior of SCL materials by a single figure of merit, namely average work. Average work is defined as the average value of a nonlinear function fitted to the friction versus normal force data, multiplied by a relevant sliding distance. The friction force is described as a function of the contact area, as derived from the elastic foundation model (EFM) as presented by Rennie et al. [19] and the interfacial shear stress. The transition from CoF to work not only circumvents the necessity for a linear relationship between the lateral and normal forces, but also represents the energy consumed when two interfaces slide over each other. To illustrate the work concept from an eyelid-SCL perspective, three commercial SCL materials, which have previously been reported to have different $\mathrm{CoF}$ under similar conditions, were tested. From low to high $\mathrm{CoF}$, the three lens materials were senofilcon A (silicone hydrogel), etafilcon A (hydrogel), and lotrafilcon B (silicone hydrogel) [13]. The lenses were characterized by means of microtribometry, using a modified version of the experimental set-up described by Roba et al. [13], which allowed for accurate determination of the contact area. The sliding work expended on the SCL was compared to that on a rabbit cornea, which acted as a model for the in-eye situation in the absence of a SCL. A classification of the frictional properties of SCL in terms of average work, or energy consumed over a certain sliding distance, allows the cumulative study of influences that external parameters (wear time, aging conditions, cleaning procedures) exert on the tribological behavior of SCL.

\section{Materials and Methods}

\subsection{Tribometer Set-Up}

The tribological experiments were conducted based on a modified version of the set-up presented by Roba et al. [13]. In brief, tribological data were recorded during reciprocal movement between a SCL mounted on a rounded sample holder ("base curve") (cyclo olefin polymer, Johnson \& Johnson Vision Care Inc, USA), matching its internal radius of curvature, and a biomimetic, coated glass surface (see below). Data collected on the cornea used the same set-up; however, the base curve had a smaller radius of curvature, and was fabricated from poly (dimethylsiloxane) (PDMS) (Sylgard 184, 1:10 mass ratio crosslinker, Dow Corning, MI, USA). Measurements were taken by means of a micro-tribometer (Basalt Must, Tetra, Germany). Cantilevers (Tetra, Germany) had a normal spring stiffness of approximately $15-16 \mathrm{~N} / \mathrm{m}$, and tangential spring stiffness between 12 and $15 \mathrm{~N} / \mathrm{m}$.

The counter-surface consisted of a 5-mm-diameter glass plate (cover slip thickness \# 1, Menzel-Gläser, Germany), which was attached with a cyanoacrylate-based glue 
(Precision Nail Glue, Kiss Products, NY, USA) to the end of a 10-mm-long glass rod of diameter $1 \mathrm{~mm}$ and mounted distally on the cantilever. Prior to fixation onto the cantilevers, glass disks were oxygen-plasma cleaned for $2 \mathrm{~min}$ (Nano, Tetra, Germany) and hydrophobized with hexamethyldisilazane (Alfa Aesar ${ }^{\circledR}$, Germany) from the gas phase at reduced pressure $(\sim 10 \mathrm{mbar})$ in a desiccator for $30 \mathrm{~min}$. Immediately prior to the friction tests, the glass plates were incubated in $1 \mathrm{mg} / \mathrm{ml}$ mucin (bovine submaxillary mucin, type I-S, Sigma-Aldrich ${ }^{\circledR}$, Germany) in HEPES 1 (10 mM N-2-hydroxyethylpiperazine-N-2 ethanesulfonic acid, $\mathrm{pH} 7.4, \mathrm{BDH}^{\mathrm{TM}}$, UK) solution for $30 \mathrm{~min}$, and rinsed with ultra-pure water (UPW, Milli-Q, Merck Millipore, MA, USA) to remove any non-adsorbed species.

A tear-like fluid (TLF) was used as a lubricant, as previously reported [12]. The TLF contains a mixture of proteins, mucin, and added lipids.

\subsection{Sample Preparation}

Prior to measurement, all the parts of the sample holder were cleaned with (1:1) UPW and detergent (hydrochloric acid $300 \mathrm{mmol} / \mathrm{l}$, detergent $1 \%$, Cobas Integra, Roche, Switzerland) rinsed with UPW and dried in a filtered hotair stream. The SCL were removed from the packing solution and rinsed three times with phosphate-buffered saline solution (PBS, D8662, Sigma-Aldrich, Switzerland) immediately before being centered on the rounded sample holder, which had been wetted with PBS. The sample holder was placed inside a Teflon chamber with an optical window at the bottom to allow for visualization of the contact area (see below for details). To prevent movement of the lens during testing, a silicone ring (polyvinylsiloxane, Provil Novo, Germany) was placed on top of the sample holder, forming a well for the lubricant. The SCL were covered with PBS until the start of the measurement, when PBS was replaced by TLF.

\subsection{Preparation of the Corneal Tissue}

Ten fresh corneas were obtained from a local, commercial, organic rabbit farm; the animals were bred for regular food consumption. In order to maintain the corneal tissue integrity, the eyelid was taped down immediately postmortem. The specimen was refrigerated (approximately $0{ }^{\circ} \mathrm{C}$ ) and transferred to the laboratory. Blunt dissection of the corneal tissue was conducted within $12 \mathrm{~h}$ post-mortem. The cornea was further prepared with removal of fascia tissue for immediate (max $60 \mathrm{~min}$ ) tribological measurement using Castroviejo scissors resulting in a corneal disk of at least $10 \mathrm{~mm}$. The procedure was performed aseptically and moisture was maintained using sterile PBS buffer solution.

\subsection{Determination of the Soft Contact Lens Contact Area}

In order to be able to observe the contact point between the glass plate and the SCL, the chamber used for the measurement was modified with respect to previously published work [13], see Fig. 1. An optical window was placed on the bottom of the Teflon ${ }^{\circledR}$ chamber. The lens, with the anterior side surface facing forward, was fitted onto the rounded sample holder. The borders of the sample holder were sandblasted in order to ensure better adhesion of the SCL, while the central area remained flat and optically transparent. The chamber was mounted on a support,

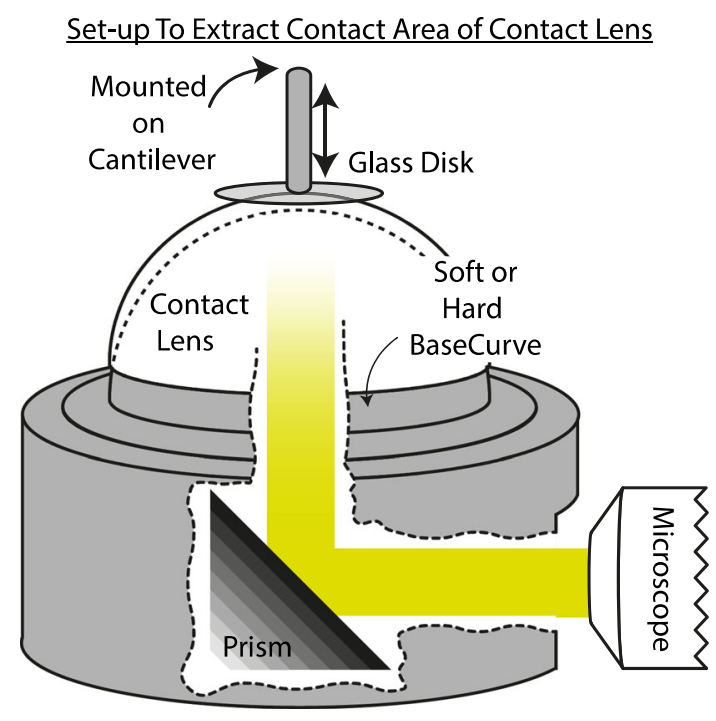

Set-up To Extract Contact Area of Rabbit Cornea
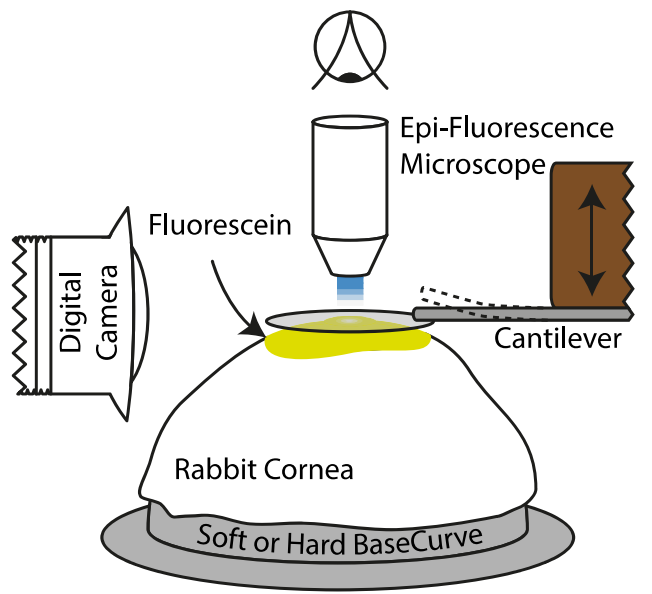

Fig. 1 (Top) Tribo set-up and contact area extraction of the contact lens. The contact area was visualized from below and defined by the appearance of Newton's rings. (Below) The set-up used to extract the contact area of the cornea. The contact area was defined from the expulsion of a fluorescent marker visualized by means of epifluorescence microscopy. The cantilever deflection was simultaneously recorded with a digital camera oriented horizontally 
having an optical prism at its base, which enabled the contact area to be characterized from below by placing a microscope (VHX 5000, Keyence, Japan or Zelos 285 M camera, Kappa, Germany attached to a Navitar Zoom 6000 objective, New York, USA) at the side of the instrument, see Fig. 1 . The contact area was obtained by fitting a circle to the region defined by the appearance of Newton's rings using image analysis software (ImageJ, http://imagej.nih. gov/ij), see Fig. 2 for an analysis example. The ability to observe the contact area allowed precise alignment of the measurement system. Perfect alignment of the SCL and glass plate is essential to maintain a constant normal pressure and to ensure conformal contact throughout the sliding cycle (no active feedback was used to correct for topographical differences). To accurately quantify the sliding work expended during a cycle, it is crucial that the normal pressure remains constant and that the sliding distance is exactly known.

\subsection{Determination of the Cornea Contact Area}

The contact area between the glass plate and the cornea model could not be observed with the methodology described for the SCL. Instead a method based on expulsion of fluorescent markers from the contact area was established, see Fig. 1. Because of its larger thickness $(0.7 \mathrm{~mm})$ compared to the SCL, (see Fig. 3), the cornea was found to wrinkle slightly on the larger-diameter base curve, which did not allow for precise measurement.

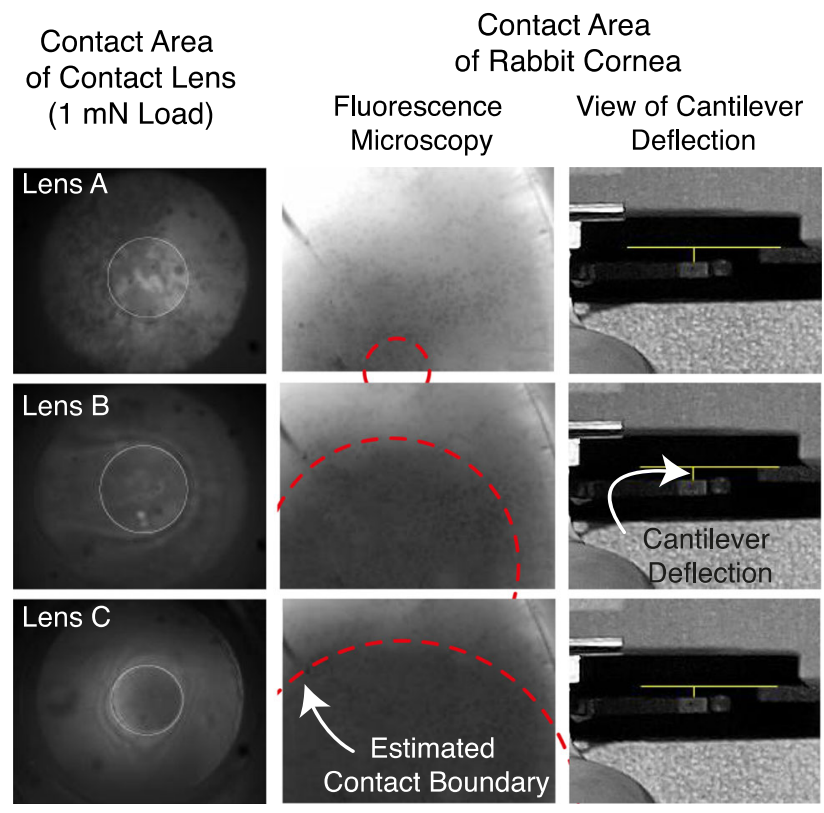

Fig. 2 Analysis example of the procedure to extract the contact area of the contact lenses (left) and cornea (right) as a function of normal load. See Fig. 1 for a description of how the contact area was visualized
Therefore, the cornea was placed either on a hard epoxy (302-3 M, Epoxy Technologies, MA, USA) or a soft PDMS (Sylgard 184, 1:10 mass ratio crosslinker, Dow Corning, MI, USA) support, with a slightly smaller radius of curvature compared to that used for the SCL. After excision, the cornea was maintained in PBS, and kept in a cold box $\left(\sim 0{ }^{\circ} \mathrm{C}\right)$ until measurement (typically no more than $4 \mathrm{~h}$ ). A drop of $0.1 \mathrm{mg} / \mathrm{ml}$ fluorescein (Polyscitech, Illinois, USA) dissolved in PBS was placed on top of the cornea. A cantilever (Tetra, Germany) with a glued glass disk (5 diameter cover slip, thickness \# 1, Menzel-Gläser, Germany) was slowly lowered onto the cornea, while the contact area was simultaneously recorded by fluorescence microscopy (FM AxioScope 2, Carl Zeiss, Germany). The applied force was measured by recording the deflection of the cantilever with a horizontally oriented digital camera (E-510, Olympus, Tokyo, Japan). Due to capillary forces, the cantilever was slightly attracted toward the cornea at zero applied force. Zero deflection (no applied force) was thus set as the maximum deflection toward the cornea in

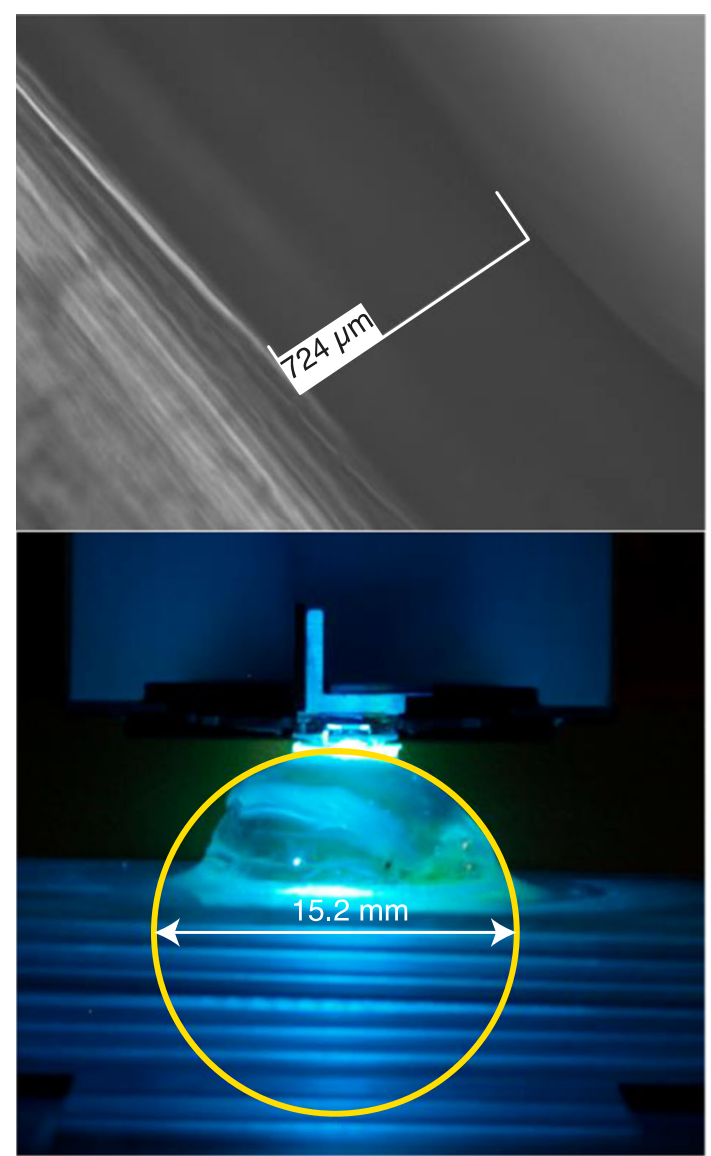

Fig. 3 (Top) A cross section of the corneal tissue as recorded by bright-field microscopy. The central thickness of the cornea was measured by image analysis. (Bottom) A side view of the corneal tissue on the sample holder. A circle was fitted to the outer borders of the cornea to determine the radius of curvature 
each series of measurements. The relative deflection was then converted into force via the known spring constant.

\subsection{Friction-Data Acquisition}

The measurement program used for the comparative friction tests involved applying three sets of seven normal forces ranging from 0.25 to $4 \mathrm{mN}$ (depending on the cantilever used). The normal loads were chosen to correspond to contact pressures that have been reported as appropriate between eyelid and cornea during a blink [30]. Since in the literature no average, overall accepted value but rather different contact pressure values, or ranges, are reported, to estimate the frictional properties of a SCL in vivo, it is also important to determine the frictional force over a relevant contact pressure interval, rather than at a single value. The friction force was recorded during two cycles, backwards and forwards, for each normal force. Only data points obtained from the second cycle were used for analysis. At the end of the two cycles, the cantilever was retracted until no contact area was visible. For the three SCL investigated, a protocol simulating short-term wear was included, consisting of two sets of 50 wear cycles performed at $2 \mathrm{mN}$ normal force between each set of seven normal forces. The wear cycles are intended to represent the effect that repeated blinking has on the frictional properties of the lens surface [13]. For the corneal tissue sample, only one ramp was chosen, as the short wear cycles induced damage of the tissue (data not shown). The measured stroke length was $1 \mathrm{~mm}$, and the sliding speed was $0.1 \mathrm{~mm} / \mathrm{s}$, in accordance with the optimized measurement procedure published by Roba et al. [13]. The entire measurement took approximately $1 \mathrm{~h}$. A speed of $0.1 \mathrm{~mm} / \mathrm{s}$ results in boundary lubrication and minimizes effects of viscous shear on the frictional force, ensuring that the experiment is representative of the frictional properties of the SCL material and not of the lubricant $[4,13]$.

For data processing, lateral and normal force values were calculated by averaging 20 data points $(2 \%$ of the stroke length) at around $0.5 \mathrm{~mm}$ sliding distance or by selecting an area where the lateral force was constant for a given normal force. The sampling frequency of the measurement was 100 points/s.

\subsection{Soft Contact Lenses}

All SCL in this study are commercially available and of the same back vertex power (-1.00 DS), see Table 1 .

\subsection{Calculation of Average Sliding Work}

\subsubsection{Choice of Model}

The sliding work was defined as friction force multiplied by a sliding distance. To assign a single number for the tribological characteristics of the sliding bodies over a relevant range of normal forces, as an alternative to a $\mathrm{CoF}$, average work was calculated. To arrive at average work, a simple function relating the friction force to the normal force was first defined. According to Rennie et al. [19], the total frictional response of an etafilcon A SCL can be divided into three separate additive components, namely visco-elastic dissipation in the bulk, interfacial shear stress, and viscous shear of the lubricant. At a sliding speed of $63 \mu \mathrm{m} / \mathrm{s}$ (comparable to the sliding speed of $0.1 \mathrm{~mm} / \mathrm{s}$ used in this work) and at low loads $(<10 \mathrm{mN})$ applied with a 1mm-diameter glass ball, the only significant contributor to the frictional force was interfacial shear stress. The frictional force, $F_{T}$, then becomes a function of the contact area, $A$, and the shear stress, $\sigma$, acting in the conformal contact:

$F_{T}=\sigma * A$

The next step was to find a relationship between the normal force and the contact area, which would allow for the shear stress to be determined from the friction force. One approach to this problem is to apply the Hertzian contact mechanics model, or one of its derivatives that take into account adhesion, such as the JKR [31] or DMT [32]

Table 1 Lenses used in this study ( -1.00 DS), together with material properties used in the EFM

\begin{tabular}{|c|c|c|c|c|c|c|c|c|}
\hline Notation & Trade name & Material & Manufacturer & Silicone? & PVP? & $\begin{array}{l}\text { Back optic zone } \\
\text { radius }(\mathrm{mm})^{\mathrm{a}}\end{array}$ & $\begin{array}{l}\text { Elastic modulus } \\
(\mathrm{MPa})[29]\end{array}$ & $\begin{array}{l}\text { Thickness } \\
(\mu \mathrm{m})^{\mathrm{b}}\end{array}$ \\
\hline Lens A & ACUVUE OASYS $^{\circledR}$ & Senofilcon A & $\begin{array}{l}\text { Johnson \& Johnson } \\
\text { Vision Care Inc }\end{array}$ & $\mathrm{Y}$ & $\mathrm{Y}$ & 8.4 & 0.7 & $76 \pm 7$ \\
\hline Lens B & ACUVUE $^{\circledR} 2$ & Etafilcon A & $\begin{array}{l}\text { Johnson \& Johnson } \\
\text { Vision Care Inc }\end{array}$ & $\mathrm{N}$ & $\mathrm{N}$ & 8.3 & 0.3 & $95 \pm 1$ \\
\hline Lens C & AIR OPTIX ${ }^{\circledR}$ AQUA & Lotrafilcon B & Alcon & $\mathrm{Y}$ & $\mathrm{N}$ & 8.6 & 1.1 & $82 \pm 4$ \\
\hline
\end{tabular}

\footnotetext{
a Manufacturer's data

b Determined by measuring the vertical distance between the focal planes at the top and bottom of the lens using white light microscopy, see supporting information $\mathrm{S} 1$
} 
models. Between a sphere and a plane, and in absence of adhesion, the circular contact area, $A_{H}$, is related to the normal force, $F_{\mathrm{N}}$, through [27]:

$A_{H}=\pi\left(\frac{3}{4} * \frac{F_{\mathrm{N}} * R}{E^{\prime}}\right)^{\frac{2}{3}}$

where $\frac{1}{E^{\prime}}=\frac{\left(1-v^{2}\right)}{E}$ is the reduced modulus at a soft-hard contact. $E$ and $v$ are the elastic modulus and Poisson ratio of the soft material, respectively, and $R$, the radius of curvature of the sphere.

However, for thin compliant materials on hard substrates, the validity of the Hertzian solution has been questioned, and the use of an elastic-foundation model (EFM) as proposed by Reedy may be more appropriate [33]. The EFM describes the layer as a Winkler surface, where the surface is modeled as a set of elastic springs without lateral interaction that upon compression deform only normally to the surface [34]. The contact area, $A_{\mathrm{EFM}}$, in an EFM problem under semi stationary conditions, as derived by Rennie et al., is given by [19]:

$A_{\mathrm{EFM}}=\pi\left(\frac{F_{\mathrm{N}} * 4 * R * t}{\pi * E^{\prime}}\right)^{\frac{1}{2}}$

The additional variable, $t$, in the EFM, is the thickness of the compliant film. Notably, the EFM results in a dependence of the contact area on the normal load of $A \propto F_{\mathrm{N}}^{1 / 2}$ as opposed to $A \propto F_{\mathrm{N}}^{2 / 3}$ in the Hertzian solution.

The sliding work, $W$, was calculated by multiplying the lateral force by the distance $\Delta x$. Combining Eqs. 1 with 3 then results in an expression for $\mathrm{W}$ as a function of $F_{\mathrm{N}}$ :

$W\left(F_{\mathrm{N}}\right)=\Delta x * \sigma * \pi *\left(\frac{4 * R * t}{\pi * E^{\prime}}\right)^{\frac{1}{2}} * F_{\mathrm{N}}^{1 / 2}$

Finally, to obtain a single value of work for each contact lens and thus enable a comprehensive comparison of lens performance, the average function of the work was calculated

$W\left(F_{\mathrm{N}}\right)_{\mathrm{avg} .}=\frac{1}{F_{\mathrm{N} 2}-F_{\mathrm{N} 1}} \int_{F_{\mathrm{N} 1}}^{F_{\mathrm{N} 2}} W\left(F_{\mathrm{N}}\right) \mathrm{d} F_{\mathrm{N}}$

for a $F_{\mathrm{N}}$ range that varies between 0.5 and $3.5 \mathrm{mN}$. This range was chosen to account for frictional drag within the contact pressure range of $0.5-18 \mathrm{kPa}$, estimated as the pressure exerted on the cornea by the upper and lower eyelid during blinking [3, 30].

\subsubsection{Data-Fitting Strategy and Error Calculation}

To account for errors in the estimation of the material properties, and random errors in experimentation, a fitting coefficient $Q$, representing the factor $\pi *\left(\frac{4 * R * t}{\pi * E^{\prime}}\right)^{\frac{1}{2}}$ in Eq. 3, was created and plotted against $N=F_{\mathrm{N}}^{1 / 2}$, to allow for simple linear regression of the experimentally determined contact area, $A_{\mathrm{EXP}}$, versus $F_{\mathrm{N}}$ :

$A_{E X P}=Q * N$

The intercept was fixed to 0 , since in the absence of adhesion (an assumption of the used form of the EFM), the contact area is zero at zero loads. The shear stress, $\sigma$, was found by simple linear regression (intercept fixed at zero) of $F_{T}$ versus $A_{\mathrm{EXP}}$ :

$F_{T}=\sigma * A_{\mathrm{EXP}}$

The average work was then calculated according to the integral in Eq. 5 after expansion:

$W_{\text {avg. }}=\frac{Q * \sigma * \Delta x}{F_{N 2}-F_{N 1}} * \frac{2}{3} *\left[F_{N 2}^{3 / 2}-F_{N 1}^{3 / 2}\right]$

The $F_{\mathrm{N}}$ range was defined as above. To derive a $95 \%$ confidence interval for the average work, $95 \%$ confidence intervals were first established for the fitting coefficients $Q$ and $\sigma$ from simple linear regression to the experimental data using technical computing software (IGOR Pro v 6.37, WaveMetrics, OR, USA). The upper and lower bounds for $\mathrm{Q}$ and $\sigma$ were used to derive the upper and lower bounds for average work, respectively.

\section{Results}

\subsection{Contact-Area Dependence on Normal Load}

The area of contact between the SCL and the glass disk was measured during the friction measurement; see Fig. 2 for a typical analysis example. For all SCL, excellent agreement was found between the EFM and the experimentally derived contact area, see Fig. 4. In contrast, the Hertzian model was found to overestimate the contact area, especially at higher normal loads. Further, as can be seen by comparing the $\mathrm{Q}$ values (representative of the SCL material properties) shown in Table 2, no changes in bulk material properties could be detected during wear cycling.

To extract the contact area of the cornea, a different methodology from that of the SCL was needed. The area was measured with fluorescence microscopy by means of the expulsion of a fluorescent marker from the contact region, while the normal force was derived from the cantilever deflection, as captured by a digital camera, see Fig. 1 for the measurement set-up and Fig. 2 for an analysis example. From the $Q$ value of the corneal surface and using the experimentally determined values for radius 
Fig. 4 Comparison between the EFM (Eq. 3) (solid line) and Hertzian (Eq. 2) (dotted line) model in predicting the experimental contact area values. For all lenses, the Hertzian model overestimates the experimentally determined contact area. Material properties used in the calculations are shown in Table 1

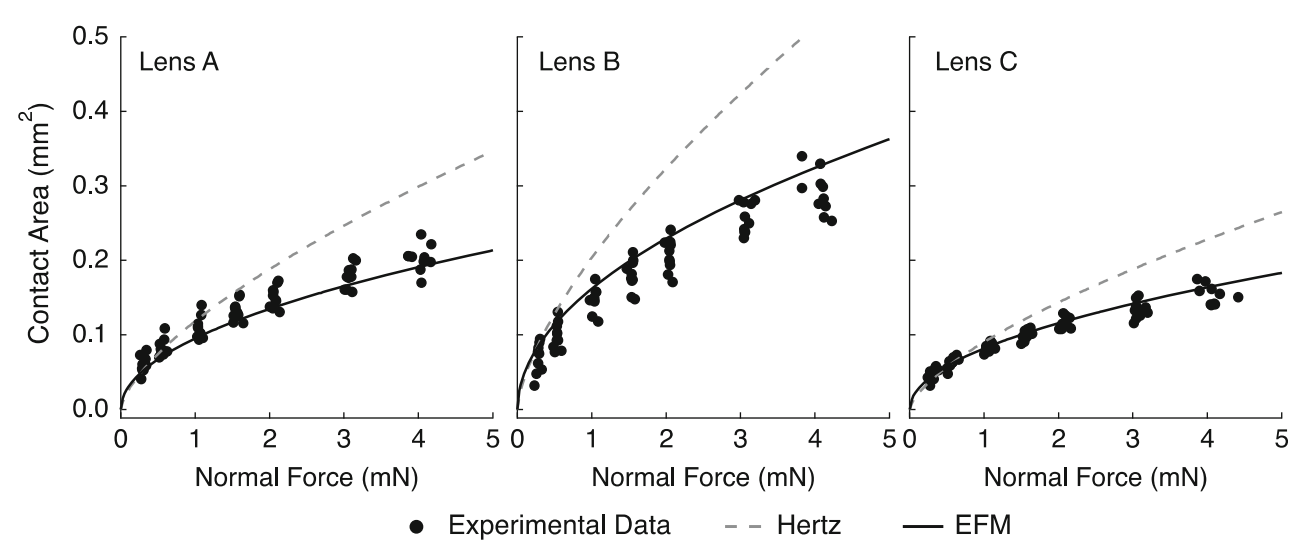

\begin{tabular}{lrllllll}
\hline Lens & Cycles & $Q$ & $\pm Q_{-} \mathrm{CI}$ & $S(\mathrm{kPa})$ & $\pm S_{-} \mathrm{CI}(\mathrm{kPa})$ & $W(\mathrm{~nJ})$ & $\pm W_{-} \mathrm{CI}(\mathrm{nJ})$ \\
\hline Lens A & 0 & 0.1038 & 0.0024 & 0.1336 & 0.0095 & 38.2 & 3.7 \\
& 50 & 0.1078 & 0.0025 & 0.1210 & 0.0089 & 35.9 & 3.5 \\
& 100 & 0.1099 & 0.0028 & 0.1214 & 0.0087 & 36.7 & 3.6 \\
Lens B & 0 & 0.1445 & 0.0037 & 0.1593 & 0.0262 & 63.4 & 12.3 \\
& 50 & 0.1431 & 0.0042 & 0.1610 & 0.0326 & 63.4 & 15.1 \\
& 100 & 0.1403 & 0.0029 & 0.2201 & 0.0577 & 85.0 & 24.5 \\
Lens C & 0 & 0.0782 & 0.0016 & 0.7539 & 0.1159 & 162.2 & 28.7 \\
& 50 & 0.0820 & 0.0021 & 1.6909 & 0.1959 & 381.7 & 55.3 \\
& 100 & 0.0813 & 0.0022 & 3.0776 & 0.2778 & 688.7 & 82.1 \\
Cornea & 0 & 2.2364 & 0.178 & 0.0104 & 0.0016 & 64.0 & 15.4 \\
\hline
\end{tabular}

Table 2 Fitting parameters $Q$ (material property factor), $S$ (interfacial shear stress) and average work $(W)$ including $95 \%$ confidence intervals (_CI) of curvature $(7.6 \mathrm{~mm})$ and thickness $(0.7 \mathrm{~mm})$ of the cornea (see Fig. 3), an elastic modulus of approximately $10.7 \mathrm{kPa}$ was found (Poisson's ratio was assumed to be 0.45). This is at least one order of magnitude lower than that of any of the SCL tested. No difference in contact area was found between the soft and the hard base curve (Fig. 5).

\subsection{Interfacial Shear Stress}

A linear relationship between the frictional force and the contact area was found for all SCLs and conditions, with the exception of lens $C$ after 100 cycles wear, see Fig. 6. For lens $\mathrm{C}$, the slope was taken as the average shear stress over the range of normal loads. The interfacial shear stress was taken as the slope between $F_{T}$ versus contact area and is tabulated in Table 2. Lens B exhibited a small, albeit not significant, increase in shear stress after 100 cycles. Lens C had a significantly $(p<0.05)$ increased average shear stress after both 50 and 100 cycles. This lens has previously been reported to have a high $\mathrm{CoF}$ under similar experimental conditions [13]. On the cornea, a shear stress approximately an order of magnitude lower than that of lens A was found (Fig. 7).

\subsection{Average Work}

The average work for all three SCL after the three different sliding cycles (i.e., 0, 50, and 100 cycles), as well as the cornea model at 0 cycles, was calculated using Eq. 8 and shown in Fig. 8. There were significant differences $(p<0.05)$, in terms of average work, between all three materials. Interestingly, only lens $\mathrm{C}$ exhibited a significant change in average work as a function of number of sliding cycles, i.e., the average work at 0 cycles $<50$ cycles $<100$ cycles. Average work on the cornea was found to correspond to the work measured on lens B; however, it was significantly higher than that measured on lens A $(p<0.05)$.

\section{Discussion}

A methodology has been presented to characterize frictional drag on soft materials in terms of work. The main advantage of using work as opposed to $\mathrm{CoF}$ is that it allows a single figure of merit to be assigned to a tribological system without requiring a linear relationship between friction and normal forces_an uncertain assumption for 

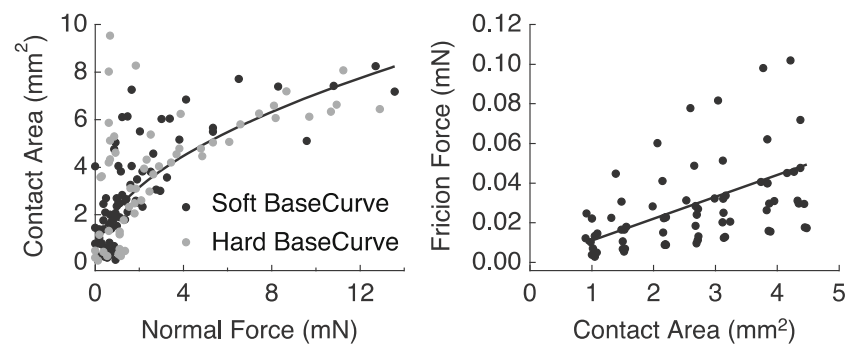

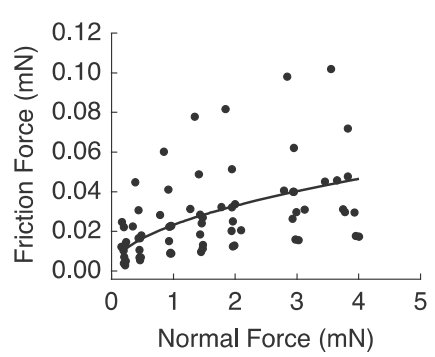

Fig. 5 (Left) Contact area of the cornea $(n=4)$ plotted against normal load as determined by fluorescence microscopy and deflection of the cantilever (see Fig. 1). (Middle) Friction force plotted versus the calculated contact area from the fit in the left graph to determine the interfacial shear stress. (Right) Friction force plotted against normal force with calculated relationship using the fitting parameters in Table 2
Fig. 6 Fitting results $(n=10)$ from $\mathrm{Ft}$ versus area (top row) to determine the interfacial shear stress and calculated relationship between $\mathrm{Ft}$ and Fn with Eq. 7 using the parameters in Table 2 (bottom row). Circles Experimental data
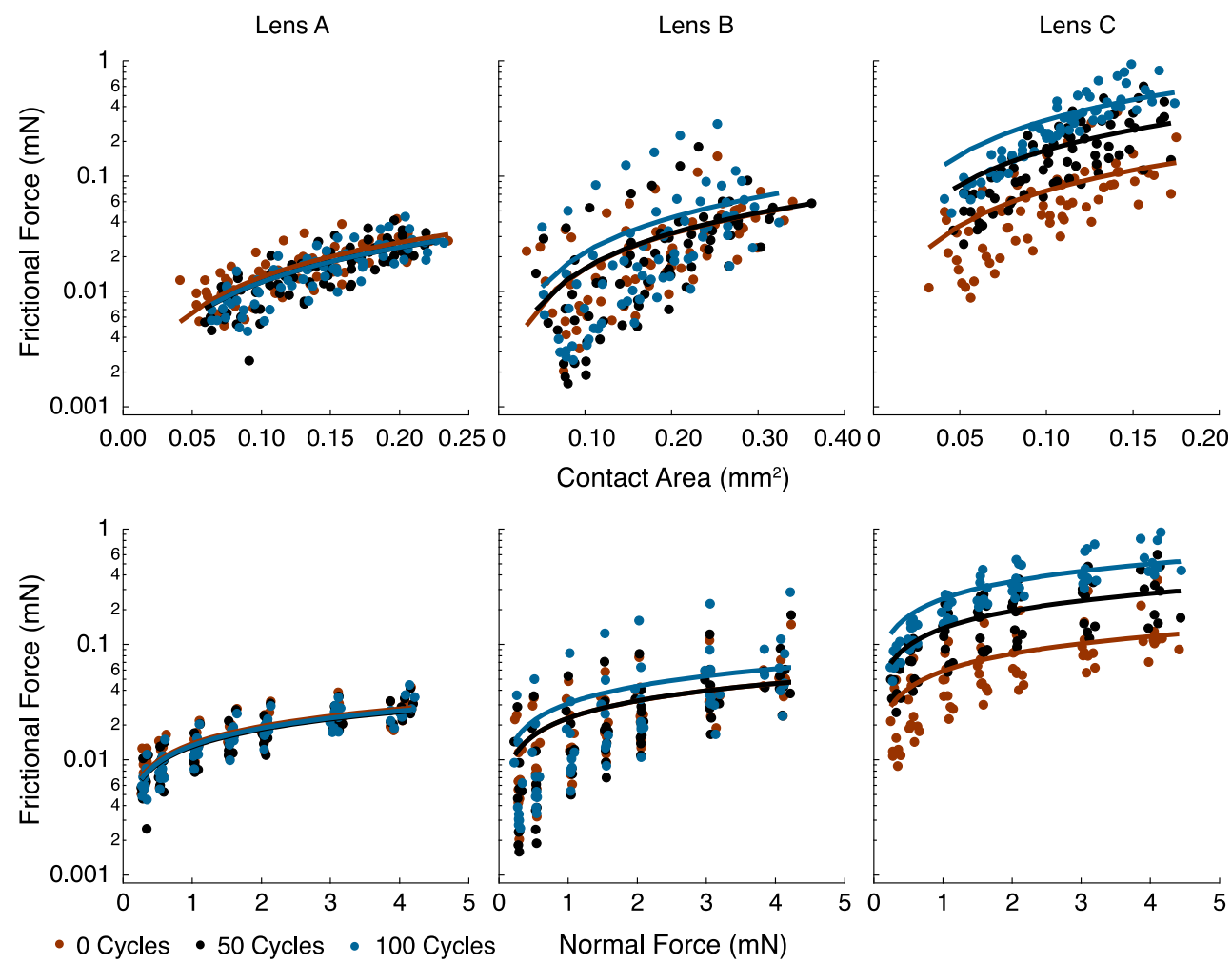

materials such as soft polymers or tissue. Another approach to distinguish between the frictional properties of the lenses in this paper could be to rely solely on interfacial shear stress. However, such an approach would lack the timedependent perspective, an important part of the work concept, which allows for cumulative measurements of expended frictional energy to be carried out. By characterizing a tribo-contact in terms of work, changes in drag that may occur over time due, for example, to wear, can be accommodated in a single figure of merit. As such, the work concept may have interesting clinical applications regarding SCL comfort where the subjective sensation of the SCL may not necessarily be correlated with the instantaneous drag between the lens and the eyelid, but to the cumulative work dissipated over the course of a day.
In the calculation of average work, it was assumed that the only contributor to the lateral force was interfacial shear stress under the experimental conditions employed. For viscoelastic materials, such as hydrogels, bulk dissipation effects can, however, also affect the friction force. A number of models have been proposed to describe friction on rubber and gel materials. Gong et al. [35] have explained the frictional response of gels at different sliding velocities from an adhesion-repulsion perspective, based on polymer scaling laws. The adhesion-repulsion model resulted in a modified Stribeck curve, with an increase in friction with speed, followed by a decrease during the elasto-hydrodynamic transition, and an increase again due to viscous shear at higher velocities [36]. At low speeds, the frictional response was described by the adhesive 


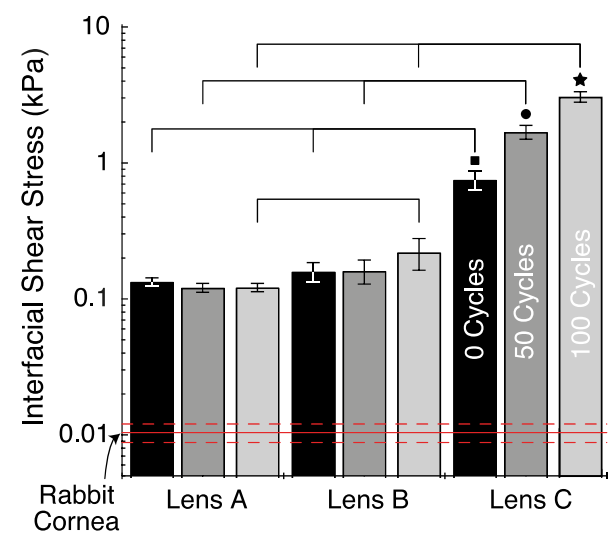

Fig. 7 Interfacial shear stress of the three contact lenses and the cornea. Error bars represent $95 \%$ confidence intervals (dotted line in the case of cornea). Lines show statistically significant differences between lenses after the same cycle time. Symbols Statistically significant differences after different cycle time of the same lens

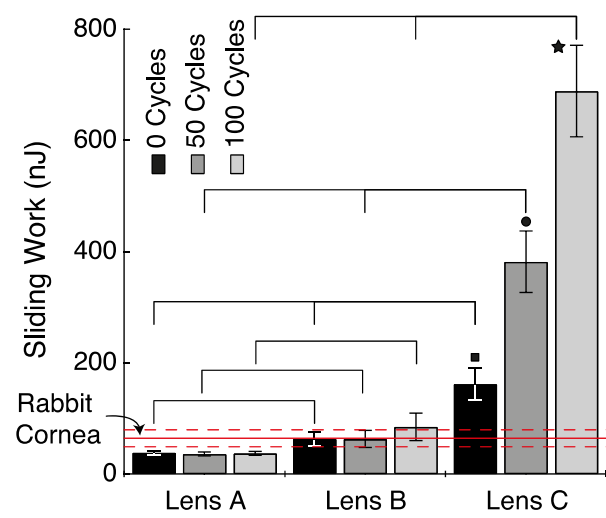

Fig. 8 Average work as calculated with Eq. 5 with a sliding distance of $2 \mathrm{~mm}$. The three lens types are plotted after 0, 50 and 100 cycles of sliding wear. The work of the cornea is plotted in for comparison. Error bar estimates represent $95 \%$ confidence intervals (dotted line in the case of the cornea). Lines show statistically significant differences between lenses after the same cycle time. Symbols Statistically significant differences after different cycle time of the same lens

interactions of the gel interfaces, resulting in interfacial shear stress. Rennie et al. applied a modified elastic foundation model based on a Winkler description of the surface that included a damping term to account for viscoelasticity. They applied the model to the study of SCL and considered the contribution of interfacial shear stress, viscoelastic dissipation and viscous shear to the friction force [19]. They found that that at low speeds $(<0.1 \mathrm{~mm} / \mathrm{s})$ and low pressures, the only significant contributor was interfacial shear stress. Scaraggi and Persson [37] recently developed a mean field theory for viscoelastic lubrication of rubber and also reported on a Stribeck curve with a local maximum when transitioning from the boundary to the elastohydrodynamic regime in the presence of a low viscosity lubricant. The explanation was an increase in the surface rolling friction, representative of viscous dissipation in the bulk. At low velocities, the interfacial shear stress was the main contributor to the frictional force. In addition, Dunn et al. [3] modeled the tribological characteristics of the eyelid as it slides over a contact lens, and using a viscosity based on natural tears reported that below $0.2 \mathrm{~mm} / \mathrm{s}$ the shear stress was constant and the sliding system was in the boundary lubrication regime. The coherence between the experimental and theoretical studies (outlined above and with respect to the contributors to the frictional force) makes it plausible that at low velocities, and low viscosity of the lubricant, the greater part of the friction force will be due to shear stress acting in the conformal contact between the glass disk and SCL or cornea.

The applicability of Eq. 7 is based on the assumption that the interfacial shear stress is load independent over the range of applied loads. In this case, a linear relationship between frictional force and contact area should be found. In order to further test whether this assumption holds for the lenses tested in this publication, line fits to the data shown in Fig. 6 were plotted on linear-linear plots together with $95 \%$ confidence intervals and residuals (see Supporting Information S3). For lens A, this relationship holds well, as represented by small and equally distributed residuals across the fitted line. Lens B show scatter in the acquired data, where some lenses deviate in their measured friction force with regard to the mean. This is likely due to a variation in the tested lenses, possibly resulting in different levels of adsorption of proteins and lipids from the TLF and a higher friction force. As a result, the residuals are biased toward negative values. For Lens $\mathrm{C}$, after 0 and 50 cycles, the situation is similar; however, after 100 cycles a systematic deviation from linearity is found in the data. This is discussed further below. Removing a series which appear to deviate from the mean response of the lenses would remedy the fitting errors to a large extent but such an action would be difficult to justify. For example, in the classification of SCL by average work, variations between lenses would be interesting to assess. Nevertheless, the data are essentially described by Eq. 7 .

Application of the EFM to the corneal contact area versus normal force curves allowed for extraction of an effective elastic modulus of the cornea (approximately $10.6 \mathrm{kPa}$ ). However, we were unable to unambiguously confirm the applicability of the EFM to the cornea, as the literature values for thickness, radius of curvature and elastic modulus show a large spread. For example, the elastic modulus of the cornea appears highly dependent on the measurement technique. Data extracted from two-dimensional strip extensiometry gave an elastic modulus of the rabbit cornea of $1.98 \mathrm{MPa}$ [38], whereas indentation studies on a rabbit cornea with atomic force microscopy 
reported only a few $\mathrm{kPa}$, depending on which corneal layer was probed [39]. In addition, Shaw et al. [40] used AFM to study the frictional properties of corneal epithelium cells and applied the EFM to extract the mechanical properties of the cells and found an effective elastic modulus of $16.5 \mathrm{kPa}$. The square-root dependence of normal load on area in the EFM has been proposed to be valid when the ratio of the thickness of the material to the radius of the counter-surface is less then 0.1 [33]. The thickness of the cornea was found to be around $700 \mu \mathrm{m}$, compared to below $100 \mu \mathrm{m}$ for the SCL. The higher thickness may thus lead to the contact area being closer to a Hertzian value, as the influence of the support becomes less important. However, the contact area was still found to vary as $F_{n}^{1 / 2}$, which is in accordance with the EFM. In addition, it should be noted that the method of obtaining the contact area versus normal force on the cornea is less precise and more prone to experimental error, compared to the SCL case where the contact area was defined by Newton's rings and the normal force measured in situ. This is mainly due to the difficulty in assigning a border defined by a fluorescence-intensity gradient and to measure a small deflection of the cantilever by image analysis. Future efforts will be targeted to attain a more precise measure of the contact area of corneal tissue. However, to support the results, we also performed indentation analysis on the cornea using a glass disk as counter-surface, fitted the data with the EFM, and found a similarly low elastic modulus of approximately $20 \mathrm{kPa}$ (see supporting information Figure S2). Therefore, during a typical friction experiment, the first tens of micrometers of the epithelium are probed and the glass disk slid over an extremely compliant surface.

In the measurements taken in this study, the average work on the cornea was found to be approximately twice that measured on lens A. This would indicate that the eyelid does less work while sliding over the lens than over the corneal epithelium. However, comparing the interfacial shear stresses, it is clear that the value on the cornea is at least one order of magnitude lower than that on lens A. In boundary lubrication, the frictional force is intimately linked to the real contact area [25]. Therefore, part of the reason for the similarity in work between the cornea and the SCL, is the extremely low elastic modulus of the cornea, which results in a contact area that is approximately an order of magnitude higher than that on the lenses. However, this is strictly a result of the contact geometry used, where a hard flat disk is pressed against a soft sphere. The contact geometry in vivo is likely to be closer to that of two soft, flat surfaces pressed against each other; consider the matching curvatures of the eyelid and the cornea, and the reported flattened epithelium that characterizes the lid wiper [41, 42]. Thus, in the eye, the effective contact area will be very similar on the cornea and on a SCL. Therefore, the frictional force is only dependent on the shear stress. If a simple assumption is made that the lid wiper is approximately $0.2 \mathrm{~mm}$ wide and $10 \mathrm{~mm}$ long, from the shear stress, the work performed by the eyelid over a $2 \mathrm{~mm}$ sliding distance across the surface of the cornea, and lenses A, B and C is approximately 50/500/1000/12,000 nJ, respectively. This highlights the importance of quantifying the contact area in addition to collecting friction and normal force data, in order to avoid over-simplified conclusions. However, among the SCL the contact area was found to vary little; therefore, the difference in terms of average work between the SCL can be representative of differences in comfort experienced in vivo.

The thought experiment above may provide some insight into how to decrease adverse strain on the eyelid epithelium during sliding over a SCL. The tested lenses were chosen on the basis of differences in bulk material as well as surface properties. Lens $\mathrm{C}$ had the highest number for average work and the highest interfacial shear stress. Lens $\mathrm{C}$ is a silicone hydrogel with a $25 \mathrm{~nm}$ plasma-generated surface layer [43], which can be assumed to result in a stiff interface that lacks sufficient mechanisms to relax applied strain. Lens B is a conventional hydrogel with low elastic modulus and a net negative charge. The combination of softness and high water content may result in an interface that is easily sheared. Lens A has a polymeric wetting agent that is believed to form dense dangling chains at the interface that can provide a lubricious plane of low shear strength, analogous to the behavior of a polymer brush and the glycocalyx. The boundary-lubricating effects of polymer brushes in good solvents are well documented in the literature [44]. Lens A is the lens with the lowest average work and interfacial shear stress. However, compared to the cornea, the interfacial shear stresses on all the lenses were significantly higher. It is hypothesized that the combination of the very soft interface and the presence of the glycocalyx, which associates with the mucin in the lubricant, is the reason for the extremely low shear stresses $(0.01 \mathrm{kPa})$ measured on the cornea. This could potentially serve as a model for the design of future SCL materials.

In the current study, a rabbit cornea acted as a model for the human counterpart. The rabbit and human cornea are of comparable dimensions [45]; however, they have been reported to differ in collagen organization in the stroma and elastic modulus [39, 46, 47]. In particular, the rabbit cornea is slightly softer than the human one. Therefore, the contact area on a human cornea is likely to be smaller under the same experimental conditions. Further, Royle et al. [48] reported on differences in glycan structure in the glycocalyx between rabbits and humans, which could potentially influence the friction properties in the boundary lubrication regime. However, the frictional forces measured on a human cornea, as reported by Wilson et al. [12], under the 
exact same conditions as applied in this work, was reported to be approximately $0.06 \mathrm{mN}$ (at $4 \mathrm{mN}$ normal load), in accordance with the results obtained herein on the rabbit cornea, see Fig. 5. As a result, it is believed that the results in terms of sliding work acquired on rabbit and human cornea are comparable. However, due to the potentially smaller contact area on the human cornea, as mentioned above, the involved shear stresses may be higher.

The contact geometry and the nature of the countersurface have a significant impact on the experimentally determined friction force. Apart from the contact geometry being different in vivo, the compliance of the interacting bodies is also different. Both the cornea and the eyelid have surfaces of very low elastic modulus - in the range of a few $\mathrm{kPa}$ - and are coated with a hydrogel-like mucus layer [5]. The frictional behavior of model hydrogel-hydrogel contacts has recently been studied by Dunn et al. [49] and was compared to the same situation where one of the sliding partners was rigid. They found a decrease in friction with speed when one of the surfaces was hard, but almost no dependence of friction on speed in the hydrogel-hydrogel "gemini" contact. Urueña et al. [50] expanded on this study and investigated the influence of sliding velocity and crosslink density of the hydrogels in a "gemini" contact on the friction force. The friction force was found to correlate with the crosslink density, which was explained by a reduced ability of the gel to relax applied shear strain with an increasing number of cross-links. At speeds up to $10 \mathrm{~mm} / \mathrm{s}$, the friction force was constant. Above this speed, the friction force increased, which was explained by an increase in the effective viscosity at the interface consisting of both the polymer chains and the fluid. The countersurface in our study was coated with a monolayer of mucin, which mimics the glycocalyx of the cornea and the eyelid, albeit with a modulus that is defined by the glass. To further mimic the in vivo situation, a soft counter-surface would be desirable. As found by Dunn et al. in a migrating contact between a hard and soft material, the friction force at a velocity of $0.1 \mathrm{~mm} / \mathrm{s}$ (used in this work) was higher than at increased velocities $(0.2-1.5 \mathrm{~mm} / \mathrm{s})$. The friction force measured with the counter-surface in our study could thus generate an overestimation of the drag between eyelid and the contact lenses in vivo. Future studies will be devoted to developing a counter-surface where the interface and the modulus more closely resemble that of the cornea-eyelid system.

It is interesting to note the deviation from linearity between friction force and contact area on lens $\mathrm{C}$ after 100 cycles. This could indicate that the area of contact is nonconformal, possibly due to an increase in the surface roughness of the lens due to testing. Lens $\mathrm{C}$ is also the stiffest of the three lenses, see Table 2, which would make the influence of surface roughness more pronounced.
Inspection of the friction versus normal force curves (Fig. 6) also shows that after 100 cycles a linear relationship between the two forces is approached. This makes surface roughness a likely cause of deviation from a linear relationship between friction and area. In addition, wear can be expected to be more severe on lens $\mathrm{C}$ as a consequence of the higher frictional forces involved during sliding.

In the calculation of average work, as discussed above, the only contributor to the friction was assumed to be interfacial shear stress. This limits the applicability of the work concept as described herein to situations where other contributions to the friction force are negligible. For example, to acquire the total energy expended by the eyelid during one blink cycle, it would be necessary to account for the different lubrication regimes that dominate at different speeds. In particular, on viscoelastic materials, in the transition from boundary-to-hydrodynamic lubrication, a speed interval may persist where bulk dissipation dominates the friction force. To account for these effects, the model would have to be extended. However, as far as a classification of SCL materials is concerned, the most relevant clinical parameter can be argued to be represented by the reported value for average work based on the observed correlation between $\mathrm{CoF}$ (acquired using similar conditions as in the present study) and comfort [10].

For soft, elastic materials, characterizing the sliding performance in terms of work instead of CoF circumvents the necessity to assume a linear relationship between frictional and normal forces. A single value of merit for work can be attained by calculating the average function of work over a range of normal forces, which can be used as a comparative measure to evaluate the sliding performance of different SCL materials. Especially, the potential to calculate cumulative work over time could provide a valuable tool to comprehensible rank the performance of different SCL materials over extended periods of wear.

\section{Conclusion}

The nonlinearity in the friction-to-normal force relationship often encountered on soft elastic materials complicates an assessment of sliding performance on the basis of a CoF. To circumvent this, a methodology has been presented that extracts the average sliding work from a nonlinear function between the lateral and normal force data. The work concept was demonstrated on three contact lens materials and a cornea. Discussing contact lens performance in terms of work opens up the possibility to calculate cumulative energy dissipation, which becomes especially useful as a comparative number if the frictional properties of the lens surface change over time, for example due to the onset of 
wear processes. Average work represents a single figure of merit on contact lens material performance, which can additionally incorporate eventual changes in lubricity occurring during wear.

Acknowledgments This study was supported by Johnson \& Johnson Vision Care Inc., Jacksonville, FL, USA.

Open Access This article is distributed under the terms of the Creative Commons Attribution 4.0 International License (http://creative commons.org/licenses/by/4.0/), which permits unrestricted use, distribution, and reproduction in any medium, provided you give appropriate credit to the original author(s) and the source, provide a link to the Creative Commons license, and indicate if changes were made.

\section{References}

1. Szczesna, D.H., Iskander, D.R.: Lateral shearing interferometry for analysis of tear film surface kinetics. Optom. Vis. Sci. 87, 513-517 (2010)

2. Wizert, A., Iskander, D.R., Cwiklik, L.: Organization of lipids in the tear film: a molecular-level view. PLoS ONE 9, e92461 (2014)

3. Dunn, A.C., Tichy, J.A., Urueña, J.M., Sawyer, W.G.: Lubrication regimes in contact lens wear during a blink. Tribol. Int. 63, 45-50 (2013)

4. Pult, H., Tosatti, S.G.P., Spencer, N.D., Asfour, J.-M., Ebenhoch, M., Murphy, P.J.: Spontaneous blinking from a tribological viewpoint. Ocul. Surf. 13, 236-249 (2015)

5. Gipson, I.K., Arguieso, P.: Role of mucins in the function of the corneal and conjunctival epithelia. Int. Rev. Cytol. 231, 1-49 (2002)

6. Muntz, A., Subbaraman, L.N., Sorbara, L., Jones, L.: Tear exchange and contact lenses: a review. J. Optom. 8, 2-11 (2015)

7. Panaser, A., Tighe, B.J.: Contact lens \& anterior eye. Cont. Lens Anterior Eye 35, 100-111 (2012)

8. Mann, A., Tighe, B.: Contact lens interactions with the tear. Exp. Eye Res. 117, 88-98 (2013)

9. Young, G., Veys, J., Pritchard, N., Coleman, S.: A multi-centre study of lapsed contact lens wearers. Ophthalmic Physiol. Opt. 22, 516-527 (2002)

10. Coles, C., Brennan, N.A.: Coefficient of friction and soft contact lens comfort. Optom. Vis. Sci. 89, e125603 (2012)

11. Kern, J., Rappon, J., Bauman, E., Vaughn, B.: Relationship between contact lens coefficient of friction and subjective lens comfort. Cont. Lens Anterior Eye 36, e26 (2013)

12. Wilson, T., Aeschlimann, R., Tosatti, S., Toubouti, Y., Kakkassery, J., Osborn Lorenz, K.: Coefficient of friction of human corneal tissue. Cornea 34, 1179-1185 (2015)

13. Roba, M., Duncan, E.G., Hill, G.A., Spencer, N.D., Tosatti, S.G.P.: Friction measurements on contact lenses in their operating environment. Tribol. Lett. 44, 387-397 (2011)

14. Luensmann, D., Jones, L.: Protein deposition on contact lenses: the past, the present, and the future. Cont. Lens Anterior Eye 35, 53-64 (2012)

15. Tosatti, S., Aeschlimann, R., Kakkassery, J., Lorenz, K.: Dynamic coefficient of friction measurements of contact lenses in tear-like fluid. Cont. Lens Anterior Eye 38, e29 (2015)

16. Nairn, J.A., Jiang, T.-B.: Measurement of the friction and lubricity properties of contact lenses. In: Proceedings of ANTEC 95, Boston (1995)

17. Kim, S.H., Marmo, C., Somorjai, G.A.: Friction studies of hydrogel contact lenses using AFM: non-crosslinked polymers of low friction at the surface. Biomaterials 22, 3285-3294 (2001)
18. Ngai, V., Medley, J.B., Jones, L., Forrest, J., Teichroeb, J.: Friction of contact lenses: silicone hydrogel versus conventional hydrogel. Tribol. Interface Eng. Ser. 48, 371-379 (2005)

19. Rennie, A.C., Dickrell, P.L., Sawyer, W.G.: Friction coefficient of soft contact lenses: measurements and modeling. Tribol. Lett. 18, 499-504 (2005)

20. Zhou, B., Li, Y., Randall, N.X., Li, L.: A study of the frictional properties of senofilcon-A contact lenses. J. Mech. Behav. Biomed. Mater. 4, 1336-1342 (2011)

21. Samsom, M., Chan, A., Iwabuchi, Y., Subbaraman, L.: In vitro friction testing of contact lenses and human ocular tissues: effect of proteoglycan 4. Tribol. Int. 89, 27-33 (2015)

22. Nguyen, D.T., Wandersman, E., Prevost, A., Le Chenadec, Y., Fretigny, C., Chateauminois, A.: Non-Amontons-Coulomb local friction law of randomly rough contact interfaces with rubber. Europhys. Lett. 104, 64001 (2014)

23. Yashima, S., Romero, V., Wandersman, E., Frétigny, C., Chaudhury, M.K., Chateauminois, A., Prevost, A.M.: Normal contact and friction of rubber with model randomly rough surfaces. Soft Matter 11, 871-881 (2015)

24. Mamada, K., Kosukegawa, H., Fridrici, V., Kapsa, P., Ohta, M.: Friction properties of PVA-H/steel ball contact under water lubrication conditions. Tribol. Int. 44, 757-763 (2010)

25. Bowden, F.P., Tabor, D.: The Friction and Lubrication of Solids. Oxford University Press, Oxford (2001)

26. Greenwood, J.A., Williamson, J.B.P.: Contact of nominally flat surfaces. Proc. R. Soc. A 295, 300-319 (1966)

27. Chaudhri, M.M., Yoffe, E.H.: The area of contact between a small sphere and a flat surface. Philos. Mag. A 44, 667-675 (1981)

28. Persson, B.N.J., Sivebaek, I.M., Samoilov, V.N., Zhao, K., Volokitin, A.I., Zhang, Z.: On the origin of Amonton's friction law. J. Phys.: Condens. Matter 20, 395006 (2008)

29. French, K.: Contact lens material properties. Part 2-mechanical behaviour and modulus. Optician 230, 29-34 (2005)

30. Shaw, A.J., Collins, M.J., Davis, B.A., Carney, L.G.: Eyelid pressure and contact with the ocular surface. Invest. Ophthalmol. Vis. Sci. 51, 1911-1917 (2010)

31. Johnson, K.L., Kendall, K., Roberts, A.D.: Surface energy and the contact of elastic solids. Proc. R. Soc. Lond. Ser. A 324, 301-313 (1971)

32. Derjaguin, B.V., Muller, V.M., Toporov, Y.P.: Effect of contact deformations on the adhesion of particles. J. Colloid Interface Sci. 53, 314-326 (1975)

33. Reedy, E.D.: Thin-coating contact mechanics with adhesion. J. Mater. Res. 21, 2660-2668 (2011)

34. Podra, P., Andersson, S.: Wear simulation with the winkler surface model. Wear 207, 79-85 (1997)

35. Gong, J., Osada, Y.: Gel friction: a model based on surface repulsion and adsorption. J. Chem. Phys. 109, 8062-8068 (1998)

36. Kurokawa, T., Tominaga, T., Katsuyama, Y., Kuwabara, R., Furukawa, H., Osada, Y., Gong, J.P.: Elastic-hydrodynamic transition of gel friction. Langmuir 21, 8643-8648 (2005)

37. Scaraggi, M., Persson, B.: Theory of viscoelastic lubrication. Tribol. Int. 72, 118-130 (2014)

38. Kling, S., Ginis, H., Marcos, S.: Corneal biomechanical properties from two-dimensional corneal flap extensiometry: application to UV-riboflavin cross-linking. Invest. Ophthalmol. Vis. Sci. 53, 5010-5015 (2012)

39. Thomasy, S.M., Raghunathan, V.K., Winkler, M., Reilly, C.M., Sadeli, A.R., Russell, P., Jester, J.V., Murphy, C.J.: Elastic modulus and collagen organization of the rabbit cornea: epithelium to endothelium. Acta Biomater. 10, 785-791 (2014)

40. Shaw, A.J., Straehla, J.P., Straehla, J.P., Collins, M.J., Limpoco, F.T., Limpoco, F.T., Davis, B.A., Dolgova, N.V., Dolgova, N.V., Carney, L.G., Keselowsky, B.G., Keselowsky, B.G., Sawyer, 
W.G., Sawyer, W.G., Perry, S.S., Perry, S.S.: Nanomechanical probes of single corneal epithelial cells: shear stress and elastic modulus. Tribol. Lett. 38, 107-113 (2010)

41. Jones, M.B., Fulford, G.R., Please, C.P., McElwain, D.L.S., Collins, M.J.: Elastohydrodynamics of the eyelid wiper. Bull. Math. Biol. 70, 323-343 (2007)

42. Knop, E., Knop, N., Zhivov, A., Kraak, R., Korb, D.R., Blackie, C., Greiner, J.V., Guthoff, R.: The lid wiper and muco-cutaneous junction anatomy of the human eyelid margins: an in vivo confocal and histological study. J. Anat. 218, 449-461 (2011)

43. Weikart, C.M., Matsuzawa, Y., Winterton, L., Yasuda, H.K.: Evaluation of plasma polymer-coated contact lenses by electrochemical impedance spectroscopy. J. Biomed. Mater. Res. 54, 597-607 (2001)

44. Klein, J., Kumacheva, E., Mahalu, D., Perahia, D., Fetters, L.J.: Reduction of frictional forces between solid surfaces bearing polymer brushes. Nature 370, 634-636 (1994)

45. Bozkir, G., Bozkir, M., Dogan, H., Aycan, K., Güler, B.: Measurements of axial length and radius of corneal curvature in the rabbit eye. Acta Med. Okayama 51, 9-11 (1997)
46. Last, J.A., Thomasy, S.M., Croasdale, C.R., Russell, P., Murphy, C.J.: Compliance profile of the human cornea as measured by atomic force microscopy. Micron 43, 1293-1298 (2012)

47. Winkler, M., Chai, D., Kriling, S., Nien, C.J., Brown, D.J., Jester, B., Juhasz, T., Jester, J.V.: Nonlinear optical macroscopic assessment of 3-D corneal collagen organization and axial biomechanics. Invest. Ophthalmol. Vis. Sci. 52, 8818-8827 (2011)

48. Royle, L., Matthews, E., Corfield, A., Berry, M., Rudd, P.M., Dwek, R.A., Carrington, S.D.: Glycan structures of ocular surface mucins in man, rabbit and dog display species differences. Glycoconj. J. 25, 763-773 (2008)

49. Dunn, A.C., Sawyer, W.G., Angelini, T.E.: Gemini interfaces in aqueous lubrication with hydrogels. Tribol. Lett. 54, 59-66 (2014)

50. Urueña, J.M., Pitenis, A.A., Nixon, R.M., Schulze, K.D., Angelini, T.E., Sawyer, W.G.: Mesh size control of polymer fluctuation lubrication mechanisms in gemini hydrogels. Biotribology 1-2, 24-29 (2015) 